\title{
Uma Metodologia Alternativa para Mensuração de Pressão Sobre o Mercado de Câmbio
}

Tito Belchior Silva Moreira

\author{
Maurício Barata de Paula Pinto \\ Geraldo da Silva e Souza
}

\author{
Departamento de Economia \\ da Universidade Católica de Brasília
}

\section{RESUMO}

Este artigo utiliza métodos estatísticos de análise multivariada para mensurar as pressões sobre os mercados cambiais de 20 países, no contexto da crise asiática de 1997/98.

Desenvolve-se um índice de pressão sobre o mercado de câmbio atribuindo pesos positivos para desvalorizações cambiais e para mudanças nas taxas de juros e pesos negativos para reduções do estoque de reservas internacionais. Os pesos são obtidos a partir de uma medida de comunalidade de cada variável utilizada na análise. Com base no índice de pressão cambial classificam-se os países da amostra por meio do método de Ward para análise de agrupamentos, identificando-se os mais atingidos pela pressão cambial iniciada em meados de 1997. A classificação obtida é validada pelo uso de análise discriminante.

\section{PALAVRAS-CHAVE}

pressão sobre o mercado de câmbio, crise asiática, análise de agrupamentos e análise discriminante

\section{ABSTRACT}

This article applies multivariate analysis to measure the exchange market pressures in 20 countries, in the context of the Asian crisis of 1997/98. We develop an index of pressure on the exchange market as a weighted average of exchange rate changes, reserve changes and interest rates changes. Changes in the exchange rate enter with positive weights, changes in reserves have negative weights and changes in interest rates have positive weights. These weights are derived from a measure of the communality of each variable used in the analysis. Using a exchange market pressure index we classify the sample, identifying by Ward's method of cluster analysis, the countries most affected by the crisis initiated in mid 1997.

The classification is validated with the use of discriminant analysis.

KEY WORDS exchange market pressure, Asian crisis, cluster analysis, and discriminant analysis

JEL Classification

F3I 


\section{INTRODUÇÃO}

A literatura econômica é prolífica na definição do conceito de pressão no mercado cambial. O enfoque modal enfatiza exclusivamente episódios com grandes e infreqüentes desvalorizações da taxa de câmbio. Uma abordagem mais abrangente é utilizada no World Economic Outlook (1999) onde a definição de pressão cambial inclui, além dos episódios de desvalorizações cambiais, os ataques especulativos que não tiveram sucesso. Neste contexto, consideram-se também os ataques à moeda nacional mesmo quando as autoridades monetárias não são forçadas a recorrer à desvalorização cambial. A manutenção da taxa de câmbio, neste caso, ocorre à custa de uma grande elevação da taxa de juros interna e/ou de perdas de reservas internacionais de proporções consideráveis. Nosso trabalho, neste artigo, diz respeito a este conceito mais amplo de pressão cambial.

Os critérios de identificação de pressão em mercado cambial podem ser divididos em qualitativos e quantitativos. A identificação qualitativa exige a caracterização de desvalorizações discretas ou mudanças de regimes cambiais. ${ }^{1}$ No critério quantitativo estabelece-se algum limite numérico para as variaçóes da taxa de câmbio, da taxa de juros e das reservas internacionais ou para um índice conjugado dessas variáveis. ${ }^{2}$

Vale destacar que em determinadas situações os indicadores quantitativos de pressão cambial apresentam limitações na caracterização de ataques especulativos, tais como: i) situação em que ataques especulativos são combatidos por meio de redução de mobilidade de capitais (centralização cambial etc.); ii) situação em que, na ocorrência de ataques especulativos, a redução do nível de reservas é compensada pela captação de recursos externos, de forma que no limite pode não haver variações no nível das reservas internacionais. Esse problema torna-se mais crítico quanto maior

1 Esse critério pode ser visto nos estudos de MELICK (L996), OTKER \& PAZARBASIOGLU (1995), GOLDBERG (L994), CUMBY \& WIJNBERGEN (L989) e BLANCO \& GARBER (1986).

2 Veja KAMINSLY \& REINHART (1997), WEYMEARK (L998) e MIRANDA (1999) para uma discussão mais detalhada a respeito das medidas de pressão sobre o mercado de câmbio. 
for o intervalo temporal em que se observam as mudanças das variáveis do índice de pressão cambial; iii) situação em que, na ocorrência de ataques especulativos, a autoridade monetária intervem no mercado cambial por meio de operações com derivativos (mercado a termo), o que é relevante inclusive no contexto da crise asiática. Em suma, qualquer intervenção do governo ou da autoridade monetária que signifique menor variação na taxa de câmbio, no sentido da desvalorização da moeda nacional, menor redução do nível das reservas internacionais e menor incremento das taxas de juros, mascara a intensidade da pressão cambial e o grau de vulnerabilidade da economia a ataques especulativos. Nesses casos, uma análise qualitativa tornar-se-ia interessante.

Pretendemos mensurar as pressões sobre os mercados cambiais no contexto da crise asiática de 1997, com base em uma amostra que inclui países do leste asiático, dentre os quais destacamos os principais protagonistas da crise (Coréia, Filipinas, Indonésia, Malásia e Tailândia), além de países sul-americanos. Nossa amostra limita-se a um conjunto de 20 países emergentes, esperando-se que o índice de pressão aqui proposto revele os principais protagonistas da crise asiática como aqueles que mais sofreram pressão cambial. ${ }^{3}$ Os países que apresentam os maiores índices (escores) são os mais atingidos pela pressão no mercado cambial. A distribuição do indicador leva à conjectura de que um índice de pressão nulo seja um ponto de referência importante: países com escores acima de zero teriam sofrido pressão no mercado de câmbio, enquanto países com escores negativos não sofreram pressão cambial.

Nossa discussão está organizada como segue. Na próxima seção é feita uma revisão da literatura. Na seção 2 apresentamos nossa abordagem metodológica para a construção do índice de pressão cambial, os critérios para análise de agrupamentos e para a validação da classificação dos países e, finalmente, destacamos as vantagens e desvantagens da metodologia ora apresentada.

3 Destaque-se que KAMINSKY \& REINHART (1999) também trabalharam com uma amostra de 20 países no referente a crises cambiais e bancárias. 
Na seção 3 são apresentados os resultados empíricos. Finalmente, a última seção é dedicada às nossas conclusões sobre o exercício econométrico levado a efeito.

\section{REVISÃO DA LITERATURA}

Os trabalhos sobre indicadores de pressão especulativa presentes na literatura decorrem de uma proposta teórica desenvolvida por Girton e Roper (1977). Estes autores avaliam o nível de intervenção no mercado de câmbio pela autoridade monetária em resposta a pressóes especulativas no mercado de câmbio.

Um importante estudo empírico sobre crises cambiais e ataques especulativos é desenvolvido por Kaminsky e Reinhart. (1999). Os referidos autores estudaram um conjunto de vinte países, no período de 1970 a 1995, e construíram um índice de pressão sobre o mercado cambial, definido como uma média ponderada das variaçóes mensais positivas da taxa nominal de câmbio e negativas das reservas internacionais. Também consideraram uma adaptação desse critério para países que passaram por períodos de inflação elevada, utilizando-se da taxa de câmbio real, de forma a não contemplar aquelas desvalorizações cambiais promovidas apenas para acompanhar a taxa de inflação.

Goldfajn e Valdés (1997) estabeleceram critérios de identificação de pressão cambial e produziram indicadores relacionados às desvalorizações do câmbio nominal e do câmbio real. Frankel e Rose (1996) definiram um colapso cambial como uma desvalorização nominal de $25 \%$ da taxa de câmbio que seja, no mínimo, 10\% maior que a taxa de desvalorização do período anterior. Goldstein, Kaminsky e Reinhart (1999) construíram um indicador de pressão cambial com base em uma média das variações da taxa de câmbio e das reservas internacionais, ajustando os dois indicadores para que estes tenham a mesma volatilidade. Em outras palavras, o indicador de crise $I$ é definido por: 


$$
I=\frac{\Delta e}{e}-\left(\frac{\sigma_{e}}{\sigma_{R}}\right) \frac{\Delta R}{R}
$$

onde $\sigma_{e}$ e $\sigma_{R}$ representam os desvios padrões das variações da taxa de câmbio e das reservas internacionais, respectivamente. Incluindo-se a variação da taxa de juros obteríamos:

$$
I=\frac{\Delta e}{e}-\left(\frac{\sigma_{e}}{\sigma_{R}}\right) \frac{\Delta R}{R}+\left(\frac{\sigma_{e}}{\sigma_{i}}\right) \frac{\Delta i}{i}
$$

Esta fórmula é semelhante à de Eichengreen et al. (1995), que construíram um índice de pressão cambial que pondera três fatores: a desvalorização cambial, a redução das reservas internacionais e a elevação abrupta das taxas de juros.

Em suma, observa-se que, tipicamente, no critério quantitativo, a literatura econômica utiliza-se de médias ponderadas de variações na taxa de câmbio, nas reservas internacionais e na taxa de juros para mensurar a pressão sobre mercado de câmbio. Nesta ordem de idéias, também utilizaremos tal critério de identificação de pressão no mercado cambial relativo ao $3^{\circ} \mathrm{e} 4^{\circ}$ trimestres de 1997, período de propagação de pressão nos mercados de câmbio dos países do leste asiático.

Faz-se mister observar aqui a importância da utilização da taxa de juros e do nível de reservas internacionais como indicadores de pressão cambial. Se essas medidas não forem consideradas, pode-se mascarar a vulnerabilidade do regime cambial, como, por exemplo, no caso de um ataque especulativo malsucedido. Uma economia só consegue sustentar um regime de câmbio fixo, dado um ataque especulativo, incrementando a taxa de juros e/ou vendendo divisas. Isto quer dizer que um indicador que meça apenas as variações da taxa de câmbio pode não capturar a vulnerabilidade daquela economia a um ataque especulativo. A magnitude do incremento da taxa de juros mede o grau de desconfiança dos agentes no concernente à capacidade de o governo manter o regime cambial fixo ou administrado. Portanto, quanto maior o incremento da taxa de juros, com o intuito de evitar um ataque especulativo, maior a vulnerabilidade do país a um colapso cambial. Esta vulnerabilidade também é revelada pela queda do nível das reservas. Um exemplo do 
funcionamento desse mecanismo é dado pelo caso do Brasil nos episódios da crise do México em dezembro de 1994 e da crise da Ásia em meados de 1997. O País conseguiu manter o regime de câmbio administrado à custa da elevação das taxas de juros e de substancial perda das reservas. Assim, se a autoridade monetária elevar a taxa de juros o suficiente para evitar fuga de capitais, um país pode manter a taxa de câmbio fixa após a incidência de um ataque contra sua moeda nacional se tiver reservas suficientes.

\section{ABORDAGEM METODOLÓGICA}

Essencialmente, nossa abordagem para a avaliação de pressão no mercado cambial envolve a caracterização de um índice unidimensional. Este tem como motivação principal a idéia central da análise fatorial, cujo modelo diz respeito à possibilidade de descrever um conjunto de $\mathrm{p}$ variáveis $\mathrm{X}_{1}, \mathrm{X}_{2, \ldots}, \mathrm{X}_{\mathrm{p}}$ em termos de um número menor de $\mathrm{m}$ fatores não diretamente observáveis. Especificamente, o modelo fatorial postula que:

$$
\mathrm{X}_{1}=a_{11} F_{1}+a_{12} F_{2}+\ldots \ldots .+a_{1 m} F_{m}+e_{i}
$$

onde $\mathrm{a}_{\mathrm{i} 1}, \mathrm{a}_{\mathrm{i} 2} \ldots \ldots . . ., \mathrm{a}_{\mathrm{im}}$ são as cargas fatoriais (pesos fatoriais ) da variável $\mathrm{X}_{\mathrm{i}}$ nos fatores $\mathrm{F}_{1}, \mathrm{~F}_{2}, \ldots \ldots . ., \mathrm{F}_{\mathrm{m}}$, respectivamente. Os $\mathrm{m}$ fatores $\mathrm{F}_{\mathrm{j}}$ são denominados fatores comuns e supõe-se que estes sejam não correlacionados, cada um com média zero e variância unitária. Os $e_{i}$ são os fatores específicos para os $\mathrm{X}_{\mathrm{i}}$. Representam medidas de erro com variância constante $\sigma_{\mathrm{i}}^{2}$, não correlacionadas entre si e com os fatores comuns.

Supondo que as variáveis têm variância unitária obtém-se:

$$
a_{11}^{2} \operatorname{var}\left(F_{1}\right)+a_{12}^{2} \operatorname{var}\left(F_{2}\right)+\ldots \ldots .+a_{1 m}^{2} \operatorname{var}\left(F_{1 m}\right)+\sigma_{1}^{2}=1
$$

e, portanto:

$$
1=a_{11}^{2}+a_{12}^{2}+\ldots \ldots \ldots+a_{1 m}^{2}+\sigma_{1}^{2}
$$


onde $\mathrm{hi}^{2}=\mathrm{a}_{\mathrm{i} 1}^{2}+\mathrm{a}_{\mathrm{i} 2}{ }^{2}+\ldots \ldots+\mathrm{a}_{\mathrm{im}}{ }^{2}$ é um termo denominado comunalidade de $X_{i}$, que representa a parte da variância de $X_{i}$ associada aos fatores comuns ou explicada por estes. A quantidade $\sigma_{\mathrm{i}}^{2}$ é também denominada de especificidade de $\mathrm{X}_{\mathrm{i}}$ e representa a parte da variância que não é relacionada com os fatores comuns. A comunalidade, portanto, é característica de cada variável. Se uma variável demonstra uma elevada comunalidade, isto significa que a variabilidade nela observada é fortemente explicada pelos fatores comuns. $^{4}$

O modelo geral de análise fatorial exposto acima tem sido usado em aplicações na psicologia, geografia, economia etc. Possui, contudo, limitações sérias, como as acentuadas em Chatfield e Collins (1980) e descritas a seguir.

i) É difícil determinar o número de fatores a serem retidos no modelo. A utilização de testes sob a hipótese de normalidade é possível para este fim, mas a convergência do processo de estimação fatorial não é garantida.

ii) As soluções $\mathrm{a}_{\mathrm{ij}}$ das cargas fatoriais, por qualquer método de estimação (extração de fatores), fixado o número de fatores comuns, não são únicas. Estimativas distintas são obtidas aplicando-se rotações ortogonais nos fatores.

iii) Não havendo solução única, torna-se subjetiva a interpretação dos fatores e também da medida da importância relativa de cada variável nestes a partir das cargas fatoriais.

É importante observar que embora os $\mathrm{a}_{\mathrm{ij}}$ possam variar em sinal e intensidade com a solução fatorial, as comunalidades $h^{2}{ }^{2}$ são constantes, isto é, são invariantes por rotaçôes ortogonais dos fatores.

Em nossa aplicação, o interesse reside em um único fator, a pressão no mercado cambial, determinante dos valores das variáveis que utilizamos. A direção da associação do fator crise com as 3 variáveis utilizadas no processo

4 Uma exposição mais detalhada sobre análise fatorial e sobre o conceito de comunalidade é apresentada em JOHNSON \& WICHERN (1992), MANLY (1994) e EVERITT \& DUNN (2001). 
de sua extração é conhecida a priori. Desse modo, pode-se definir um escore de pressão cambial utilizando-se como peso de cada variável uma medida de comunalidade relativa ajustada pela direção predeterminada. A medida relativa de comunalidade tem a vantagem de ser independente de rotações ortogonais, e pode ser aproximada por um método heurístico.

O método heurístico de aproximação da comunalidade dá-se por meio da regressão de Xi (padronizada) nas variáveis restantes $\mathrm{Xj}$ (padronizada), $\operatorname{com} j \neq i$. A razão entre a soma de quadrados explicados pela regressão, $\mathrm{SQRegr}_{i}$, e a soma dos quadrados totais nessa regressão, $\mathrm{SQT}_{i}$, é dada por $\mathrm{R}_{\mathrm{i}}^{2}$ (coeficiente de determinação da regressão). Note-se que, na realidade, $\mathrm{R}_{\mathrm{i}}^{2}$ pode ser visto como uma proxy para a comunalidade de Xi. De fato, sob a hipótese da estrutura fatorial, obtém-se:

$$
1=h_{i}^{2}+\sigma_{i}^{2}
$$

e sob a hipótese de regressão linear de $X_{i}$ nas outras variáveis tem-se que $(\mathrm{n}-\mathrm{l})=\mathrm{SQRegr} \mathrm{r}_{\mathrm{i}}+\mathrm{SQE}_{i}$, onde n é o número de observações, os resíduos têm média zero e $\mathrm{SQE}_{i}$ é a soma dos quadrados dos resíduos. Logo: $1=$ $\left(\frac{S Q \operatorname{Re} g r_{i}}{n-1}+\frac{S Q E_{i}}{n-1}\right)$. Como os fatores são determinados pelos $\mathrm{X}_{i}$ pode-se pensar no modelo de regressão como a versão observacional do modelo fatorial. Neste contexto, o termo $\left(\frac{S Q E_{i}}{n-1}\right)$ estima $\sigma_{i}^{2}$ e $\left(\frac{S Q \operatorname{Re} g r_{i}}{n-1}\right)=R_{i}^{2}$ estima $h_{i}^{2}$. Tais comentários têm natureza heurística, mas são a base do uso de $R_{i}^{2}$ como proxy para $h_{i}^{2}$, conforme Johnson e Wichern (1992, p. 410-411).

Vale destacar que no caso de uma regressão existe alguma relação de causalidade, ou condicionalidade, entre as variáveis. Daí a necessidade de especificação do modelo em termos de variáveis exógenas. No que tange à análise fatorial, esta hipótese não é necessária. Procura-se determinar uma relação de interdependência entre as variáveis, expressas por fatores comuns não observáveis, havendo apenas a condição necessária de existência de alguma relação teórica entre as variáveis. A descrição desta relação encontra-se formalizada em Johnson e Wichern (1992, p. 343-347). 
Definimos como indicador agregado de crise cambial a quantidade:

$$
I=\sum_{i=1}^{p} \gamma_{i} \Psi_{i} \mathrm{X}_{i}
$$

onde $p=3, \gamma_{\mathrm{i}}$ é a direção ou sinal estabelecido a priori para $\mathrm{X}_{\mathrm{i}} \mathrm{e}$

$$
\Psi_{i}=\frac{R_{i}^{2}}{\sum_{j=1}^{p} R_{j}^{2}}
$$

é a comunalidade relativa.

O indicador $I$ é semelhante a um fator geral no modelo fatorial. Outra analogia seria definida pelo escore do primeiro componente na análise de componentes principais. (MANLY, 1994). Os valores de I permitem ordenar os elementos da amostra, países em nossa aplicação, segundo a intensidade com que respondem a esta medida agregada de pressão cambial.

A disponibilidade da medida agregada de crise $I$ leva naturalmente à investigação sobre a existência de grupos homogêneos, i.e, de conglomerados de países com valores similares do escore. Nossa abordagem neste aspecto se utiliza do método de classificação de Ward (EVERITT \& DUNN, 2001) e da distância euclidiana como medida de proximidade entre grupos. A técnica de Ward é um método de classificação hierárquico que procura formar agrupamentos com a menor variabilidade possível dentro dos grupos. O critério tem um princípio similar ao da análise de variância multivariada. Nossa escolha formal do número de estratos de pressão cambial baseou-se nas estatísticas comumente utilizadas na análise de agrupamentos (Critério de Agrupamento Cúbico e Quadrado de Correlação Múltipla). ${ }^{5}$ A classificação dicotômica gerada pelo processo foi validada com o uso de análise discriminante. (EVERITT \& DER, 1996). Nestes termos, o interesse reside na probabilidade posterior de classificação dos países em cada grupo e na proporção de países erroneamente classificados.

5 Veja EVERITT \& DER (1996). 
Para organizar a apresentação resumimos os principais resultados da presente seção e antecipamos alguns resultados das seções seguintes. Assim, utilizamos métodos estatísticos de análise multivariada para mensurar as pressões sobre os mercados cambiais de 20 países, no contexto da crise asiática de 1997/98. Desenvolvemos um índice de pressão cambial atribuindo pesos positivos para desvalorizações cambiais e para mudanças nas taxas de juros e pesos negativos para reduçóes do estoque de reservas internacionais. Os pesos são obtidos a partir de uma medida de comunalidade de cada variável utilizada na análise. Portanto, o índice de pressão é formado com base em uma média ponderada. Com base no índice mencionado, classificamos os países da amostra em 2 grupos: um formado pelos países que sofreram maior pressão no mercado cambial durante o $3^{\circ}$ terceiro e $4^{\circ}$ trimestres de 1997 e, o outro, que apresenta pouca ou nenhuma instabilidade no mercado de câmbio no mesmo período.

\subsection{Vantagens e Desvantagens dos Diferentes Métodos de Mensuração de Pressão Cambial}

O indicador de pressão no mercado de câmbio é definido a partir de uma média ponderada cujos pesos das variáveis são fixados por meio de medidas de comunalidade. O conceito de comunalidade é herdado dos modelos de análise fatorial. Nossa abordagem difere dos métodos tradicionais na definição do sistema de pesos e no processo de padronização das variáveis componentes do indicador de crise.

$\mathrm{O}$ indicador de pressão no mercado cambial desenvolvido neste artigo tem semelhanças e diferenças marcantes em relação ao apresentado por Goldstein, Kaminsky e Reinhart (2000). Ambas as abordagens procuram padronizar as variáveis e definem o indicador de crise como uma combinação linear das variáveis disponíveis. Em ambos os casos procura-se uma padronização tal que todas as variáveis do estudo apresentem a mesma volatilidade. A diferença fundamental está no processo de padronização. Goldstein et al. (2000) escolhem um nível de volatilidade arbitrário e os pesos das variáveis são definidos em termos desta volatilidade básica. Nosso indicador, por outro lado, utiliza a média como ponto de referência, usa padronização unitária, e o sistema de pesos é definido pela intensidade da correlação de 
cada variável com o fator pressão cambial. Deste modo, acreditamos ter obtido um construto para o indicador de pressão cambial que não somente padroniza as variáveis de modo independente como também leva em conta a importância relativa das variáveis no construto de interesse na avaliação da pressão no mercado cambial.

Um outro aspecto importante a ser destacado é que a maioria dos indicadores de pressão cambial apresentados na literatura é calculada, para cada país em separado, com periodicidade definida (mensal ou trimestral, por exemplo). Assim, os pesos que ponderam as variações nas taxas de câmbio, reservas internacionais e taxas de juros são estimados a partir de séries de tempo.

Por outro lado, no indicador aqui proposto a avaliação da pressão especulativa em um determinado país não está dissociada da pressão especulativa de outros países, pois os pesos são calculados a partir de uma regressão em cross-section. Considerando que principalmente a partir da década de 90 observa-se: i) uma tendência crescente de integração dos mercados de capitais; ii) uma maior mobilidade de capital; iii) uma maior liberalização financeira e; iv) uma maior abertura das contas de capitais, é natural admitir-se que as pressões no mercado de câmbio de um determinado país afetam e pressionam os mercados cambiais de outros países. Há uma vasta literatura debatendo o contágio proveniente de crises cambiais entre países. O exemplo mais citado na literatura sobre contágio diz respeito à crise asiática de 1997, que se caracterizou por um grau de propagação entre os países emergentes até então sem precedentes na história das crises cambiais.

Em vista disso, acreditamos ter obtido um construto para o indicador de pressão cambial que leva em conta a interdependência e o efeito contágio entre os mercados cambiais dos países emergentes na avaliação de pressão nos mercados de câmbio.

Vale destacar ainda que para o estudo de pressão cambial para apenas um país, ou mesmo um pequeno grupo de países, a metodologia de Goldstein, Kaminsky e Reinhart (2000) é a mais apropriada. Entretanto, para um estudo abrangendo um grande número de países a metodologia por nós apresentada é a mais apropriada por ser mais parcimoniosa. Utilizando-se 
a metodologia de Goldstein et al. (2000) seria necessário construir um indicador de pressão cambial para cada país. Utilizando a nossa metodologia, construímos apenas 2 indicadores, um para o $3^{\circ}$ trimestre de 1997 e um outro para o trimestre seguinte.

\section{RESULTADOS EMPÍRICOS}

A Tabela Al, apresentada no Apêndice, relaciona os valores das seis variáveis utilizadas em nossa análise na forma original (não padronizadas), onde: $\mathrm{X}_{1}=$ Razão da taxa de câmbio nominal do $3^{\circ}$ trimestre de 1997 em relação ao trimestre anterior; $X_{2}=$ Razão da taxa de câmbio nominal do $4^{\circ}$ trimestre de $1997 \mathrm{em}$ relação ao trimestre anterior; $\mathrm{X}_{3}=$ Razão do nível de reservas internacionais do $3^{\circ}$ trimestre de 1997 em relação trimestre anterior; X4 = Razão do nível de reservas internacionais do $4^{\circ}$ trimestre de 1997 em relação trimestre anterior; X5 = Razão da taxa de juros nominal sobre depósitos do $3^{\circ}$ trimestre de 1997 em relação ao trimestre anterior; X6 = Razão da taxa de juros nominal sobre depósitos do $4^{\circ}$ trimestre de 1997 em relação ao trimestre anterior.

Como as pressões sobre os mercados cambiais das economias do leste asiático iniciaram-se em Julho de 1997 e se propagaram com mais força até o final desse ano, optamos por trabalhar com índices do $3^{\circ} \mathrm{e} 4^{\circ}$ trimestres de 1997.

A Tabela $\mathrm{l}$ apresenta proxies $\left(\mathrm{Hi}^{2}\right)$ para as comunalidades absolutas, comunalidades relativas $\left(\Psi_{i}\right)$ e direções de crise $(\gamma \mathrm{i})$ das seis variáveis determinantes do indicador $I$ :

TABELA 1 - COMUNALIDADES ABSOLUTAS E RELATIVAS E RESPECTIVOS SINAIS: $3^{\circ}$ E $4^{\circ}$ TRIMESTRES DE 1997

\begin{tabular}{lccccccc}
\hline $\begin{array}{l}\text { Variáveis } \\
\text { 30 Trimestre }\end{array}$ & $\mathrm{Hi}^{2}$ & $\Psi_{i}$ & $Y_{i}$ & $\begin{array}{c}\text { Variáveis } \\
\text { 4 Trimestre }\end{array}$ & $\mathrm{Hi}^{2}$ & $\Psi_{i}$ & $Y_{i}$ \\
\hline$X_{1}$ & 0,341 & 0,470 & + & $X_{2}$ & 0,445 & 0,440 & + \\
$X_{3}$ & 0,090 & 0,130 & - & $X_{4}$ & 0,482 & 0,470 & - \\
$X_{5}$ & 0,288 & 0,400 & + & $X_{6}$ & 0,095 & 0,090 & + \\
Total & 0,719 & 1,000 & & Total & 1,022 & 1,000 & \\
\hline
\end{tabular}


Os indicadores de pressão no mercado de câmbio para o $3^{\circ}$ e $4^{\circ}$ trimestres são explicitados da seguinte forma:

$$
\begin{aligned}
& \mathrm{I}\left(3^{\circ} \text { trimestre }\right)=\Psi_{1} \mathrm{X}_{1}-\Psi_{3} \mathrm{X}_{3}+\Psi_{5} \mathrm{X}_{5} \\
& \mathrm{I}\left(4^{\circ} \text { trimestre }\right)=\Psi_{2} \mathrm{X}_{2}-\Psi_{4} \mathrm{X}_{4}+\Psi_{6} \mathrm{X}_{6}
\end{aligned}
$$

onde, ressaltamos uma vez mais, os Xi são padronizados.

Merece ser ressaltado que o indicador de pressão cambial aqui proposto, assim com qualquer indicador proposto pela literatura, pode ter a periodicidade ajustada a depender da conveniência do estudo. Obviamente, períodos mais curtos (mensais, por exemplo) capturam melhor as variações nas taxas de câmbio, nas reservas internacionais e nas taxas de juros. A escolha da periodicidade trimestral decorre de mera conveniência para o nosso propósito. Como a pressão nos mercados cambiais dos países do leste asiático ocorreu principalmente ao longo do segundo semestre de 1997, dados trimestrais tornam o indicador de pressão mais parcimonioso. Caso optássemos por uma periodicidade mensal, por exemplo, teríamos que calcular e analisar 6 indicadores. Da mesma forma, o indicador aqui proposto poderia trabalhar, por exemplo, com apenas duas variáveis: taxa de câmbio e reservas internacionais. A escolha das variáveis para compor o indicador de pressão cambial também depende da conveniência do estudo.

A Tabela 2, apresentada a seguir, indica os países da amostra com os seus respectivos escores, ordenados em ordem crescente, para o $3^{\circ}$ e $4^{\circ}$ trimestres. Os países com maiores escores foram os mais atingidos pela pressão no mercado cambial. A distribuição do indicador leva à conjectura de que $I=0$ seja um ponto de referência importante: sugere que países com escores acima de zero sofreram pressão no mercado de câmbio, enquanto países com escores negativos não sofreram pressão cambial. 
TABELA 2 - ESCORES DOS PAÍSES: INDICADOR DE PRESSÃO CAM$B L A L$

\begin{tabular}{llll}
\hline \multicolumn{1}{c}{$3^{\circ}$ Trimestre de 1997} & \multicolumn{2}{c}{$4^{0}$ Trimestre de 1997} \\
\hline México & $-1,10$ & Argentina & $-1,06$ \\
Sri Lanka & $-0,74$ & China & $-0,90$ \\
Jordânia & $-0,70$ & Jordânia & $-0,90$ \\
China & $-0,58$ & México & $-0,73$ \\
Peru & $-0,52$ & Peru & $-0,71$ \\
Argentina & $-0,51$ & Hong-Kong & $-0,69$ \\
Brasil & $-0,49$ & Polônia & $-0,65$ \\
Coréia & $-0,37$ & Sri-Lanka & $-0,42$ \\
Hong-Kong & $-0,23$ & Venezuela & $-0,34$ \\
Venezuela & $-0,23$ & Chile & $-0,29$ \\
Cingapura & $-0,20$ & Rep. Tcheca & $-0,18$ \\
Rep. Tcheca & $-0,16$ & Colômbia & 0,02 \\
Colômbia & $-0,13$ & Cingapura & 0,05 \\
Chile & 0,04 & Turquia & 0,33 \\
Polônia & 0,05 & Brasil & 0,46 \\
Malásia & 0,49 & Tailândia & 0,60 \\
Turquia & 0,55 & Malásia & 0,68 \\
Filipinas & 1,37 & Filipinas & 1,10 \\
Indonésia & 1,44 & Coréia & 1,79 \\
Tailândia & 2,03 & 1,84 \\
\hline
\end{tabular}

Nota: Maior escore indica maior pressão cambial.

\subsection{Análise de Agrupamento}

A conjectura de que $I=0$ seja um ponto de corte na pressão cambial é praticamente confirmada pela análise de agrupamentos.

Os resultados da análise de agrupamento são apresentados na Tabela 3, a seguir, onde os valores de $\mathrm{R}^{2}$ e CCC sugerem 2 estratos ou grupos. 
TABELA 3 - ANÁLISE DE AGRUPAMENTO: INDICADOR DE PRESSÃO CAMBIAL

\begin{tabular}{|c|c|c|c|c|c|}
\hline $\begin{array}{l}\mathrm{N}^{\circ} \text { de } \\
\text { Conglomerados } \\
3^{0} \text { Trimestre }\end{array}$ & $\mathrm{R}^{2}$ & $\mathrm{CCC}$ & $\begin{array}{c}\mathrm{N}^{\circ} \text { de } \\
\text { Conglomerados } \\
4^{0} \text { Trimestre }\end{array}$ & $\mathrm{R}^{2}$ & $\mathrm{CCC}$ \\
\hline 19 & 1,00000 & . & 19 & 1,00000 & . \\
\hline 18 & 1,00000 & . & 18 & 0,99999 & . \\
\hline 17 & 0.99999 & . & 17 & 0,99995 & . \\
\hline 16 & 0.99996 & . & 16 & 0,99991 & . \\
\hline 15 & 0.99992 & . & 15 & 0,99982 & . \\
\hline 14 & 0.99987 & . & 14 & 0,99973 & . \\
\hline 13 & 0.99981 & . & 13 & 0,99954 & . \\
\hline 12 & 0.99966 & . & 12 & 0,99931 & . \\
\hline 11 & 0.99946 & . & 11 & 0,99879 & . \\
\hline 10 & 0.99913 & . & 10 & 0,99819 & . \\
\hline 9 & 0.99859 & . & 9 & 0,99697 & . \\
\hline 8 & 0.99702 & . & 8 & 0,99543 & . \\
\hline 7 & 0.99109 & . & 7 & 0,99115 & . \\
\hline 6 & 0.98467 & . & 6 & 0,98300 & . \\
\hline 5 & 0.93064 & . & 5 & 0,97185 & . \\
\hline 4 & 0.90947 & $-2,7090$ & 4 & 0,95251 & $-0,570$ \\
\hline 3 & 0.86571 & $-1,6550$ & 3 & 0,86617 & $-1,643$ \\
\hline 2 & 0.74654 & $-0,5860$ & 2 & 0,72419 & $-0,946$ \\
\hline 1 & 0.00000 & 0,0000 & 1 & 0,00000 & 0,000 \\
\hline
\end{tabular}

Nota: $\mathrm{R}^{2}=$ Quadrado da Correlação Múltipla, $\mathrm{CCC}=$ Critério de agrupamento cúbico.

Os países classificados no grupo 2 apresentam os maiores escores e, portanto, são identificados como os que mais sofreram pressão cambial, conforme se segue:

Para o $3^{\circ}$ trimestre de 1997 temos a seguinte classificação:

Grupo 1 - Argentina, Brasil, Cingapura, Chile, China, Colômbia, Coréia, Hong-Kong, Jordânia, Malásia, México, Peru, Polônia, República Tcheca, Sri Lanka, Turquia, e Venezuela. 
Grupo 2 - Filipinas, Indonésia e Tailândia.

Para o $4^{\circ}$ trimestre de 1997 temos a seguinte classificação:

Grupo 1 - Argentina, Cingapura, Chile, China, Colômbia, Hong-Kong, Jordânia, México, Peru, Polônia, República Tcheca, Sri Lanka, e Venezuela.

Grupo 2 - Brasil, Coréia, Filipinas, Indonésia, Malásia, Tailândia e Turquia.

Entre julho e dezembro de 1997 a instabilidade no leste asiático pressionou os mercados de câmbio da maioria dos países emergentes em todo o mundo. A seguir apresentamos a cronologia da crise (BIS, 1998 e TREUHERZ, 2000), enfatizando os fatos estilizados dos mercados cambiais dos principais protagonistas da crise (Coréia, Filipinas, Indonésia, Malásia e Tailândia).

- A pressão no mercado cambial dos países do leste asiático iniciou-se em 2 de julho de 1997 com a desvalorização de 10\% da moeda tailandesa, o Bath.

- As Filipinas foram o primeiro vizinho a sucumbir à pressão especulativa, deixando de lado o seu câmbio indexado ao dólar em 11 de julho de 1997. Nesta mesma data, os limites da banda cambial da moeda indonésia, Rupiah, foi alargado de $8 \%$ para $12 \%$.

- A moeda da Malásia, Ringgit, desvalorizou-se em 4,8\% no final de julho.

- O Rupiah da Indonésia flutuou em 14 de agosto de 1997.

- O FMI aprova um crédito de U\$ 3,9 bilhões para a Tailândia em 20 de agosto de 1997.

- Entre 20 e 23 de outubro aumenta a pressão sobre a moeda coreana, Won.

- Em 31 de outubro o Banco Central do Brasil aumenta a taxa de juros para 43\% depois de intensa pressão sobre o Real.

- Em 5 de novembro o FMI aprova um crédito de U\$ 10,1 bilhões para a Indonésia, sendo que U\$ 3 bilhões foram disponibilizados imediatamente. 
- Em 20 de Novembro a flutuação da banda diária do Won koreano alargou-se de $\pm 2 \frac{1}{4} \%$ para $\pm 10 \%$.

- Em 4 de Dezembro o FMI aprova um crédito de U\$21 bilhóes para os próximos 3 anos, sendo que U\$ 5,6 bilhões foram disponibilizados imediatamente.

- Em 16 de dezembro de 1997 o Won koreano flutuou.

- Em dezembro de 1997 houve uma forte desvalorização do Ringgit, da Malásia. As tentativas do Banco Central da Malásia de evitar a desvalorização resultou em perdas de reservas internacionais.

A classificação formal da análise de agrupamento inclui os principais protagonistas da crise do leste asiático no Grupo 2 , tanto no $3^{\circ}$ trimestre de 1997 (Filipinas, Indonésia e Tailândia) quanto no $4^{\circ}$ trimestre do mesmo ano (Brasil, Coréia, Filipinas, Indonésia, Malásia, Tailândia e Turquia), destacando-se também a classificação do Brasil e Turquia neste último trimestre. No Grupo 2 foram classificados os países que sofreram significativa pressão no mercado de câmbio, enquanto as economias que compóem o Grupo 1 são aquelas que não foram significativamente atingidas pela instabilidade cambial deflagrada no leste asiático a partir de meados de 1997.

Vale destacar que no $4^{\circ}$ trimestre de 1997 a Turquia e o Brasil apresentaram os menores escores do grupo 2, conforme Tabela 2, o que indica que sofreram menor pressão cambial que os principais protagonistas da crise. $\mathrm{O}$ indicador de pressão de mercado de câmbio, ora apresentado, caracterizou, no caso do Brasil, um caso típico de ataque especulativo malsucedido. As autoridades monetárias brasileiras conseguiram manter o regime de bandas cambiais sob controle, mas à custa de perdas de reservas internacionais e de elevação das taxas de juros, conforme dados da Tabela Al. No caso da Turquia, houve desvalorização cambial e queda das reservas internacionais, conforme Tabela Al. Neste caso, ambos os países revelaram-se vulneráveis aos choques externos. 


\subsection{Análise Discriminante}

A adequação da classificação dos países é investigada adicionalmente por meio de análise discriminante, com o uso da função linear discriminante de Fisher. (JOHNSTON \& WICHERN, 1992). Esta abordagem não produziu erros de classificação, quer se considere o indicador $I$ ou as variáveis originais em suas dimensões marginais. As Tabelas A2 e A3, anexas, mostram os resultados obtidos com $I$.

Também utilizamos a análise discriminante não-paramétrica. O método não-paramétrico é descrito em Conover (1999), que propõe classificar as observações por meio da aplicação das transformações de ranks. Cada variável é ordenada separadamente, e a função discriminante linear também é computada sobre ranks. Dessa forma, pode-se averiguar se os países foram corretamente classificados pela análise discriminante, independentemente de suas hipóteses distribucionais. Usualmente a análise discriminante é levada a efeito sob a hipótese de distribuição normal multivariada para os dados, embora seja esta uma distribuição pouco comum. A abordagem não-paramétrica é então comparada com esta. Discrepâncias de resultados são indicativos de não normalidade. Para a base de dados usada neste artigo não houve grandes discrepâncias entre os resultados das duas análises. Apenas dois, dentre vinte países, foram erroneamente classificados tanto no $3^{\circ}$ quanto no $4^{\circ}$ trimestre de 1997. No $3^{\circ}$ trimestre de 1997, Chile e Malásia foram classificados na análise discriminante normal no Grupo 1 e reclassificados no grupo 2 com o método não-paramétrico, conforme Tabela $\mathrm{A} 4$, anexa. No $4^{\circ}$ trimestre de 1997, Cingapura e República Tcheca também foram classificados na análise discriminante normal no Grupo 1 e reclassificados no grupo 2 com o método não-paramétrico (Tabela A5).

\subsection{Indicador de Pressão Cambial a la Goldstein, Kaminsky e Reinhart}

Para fins de comparação entre a metodologia aqui apresentada e a de Goldstein, Kaminsky e Reinhart (2000) relacionamos, na Tabela 4, os escores de pressão cambial da metodologia proposta por estes autores. Foram consideradas variações trimestrais entre o $4^{\circ}$ trimestre de 1995 e o último 
trimestre de 1997. Este período compreende tempos de tranqüilidade até o $2^{\circ}$ trimestre de 1997, e tempos de turbulência nos mercados cambiais. O indicador de pressão cambial é definido conforme a equação 1.2 e os resultados mostram que nenhum país sofreu pressão cambial no $3^{\circ}$ trimestre de 1997. Por outro lado, o Brasil, a Indonésia e Cingapura foram classificados no grupo dos países que sofreram pressão cambial no $4^{\circ}$ trimestre de 1997. Aqueles países que obtiveram escores superiores ao valor de dois desvios padrões adicionado ao valor da média do indicador são classificados como aqueles que sofreram pressão no mercado de câmbio.

TABELA 4 - ESCORES DOS PAÍSES: INDICADOR DE PRESSÃO CAMBIAL A LA GOLDSTEIN, KAMINSKY E REINHART

\begin{tabular}{llccc}
\hline Países & Indicador de crise $(\mathrm{I})$ & $\bar{I}+2 \sigma$ & $\begin{array}{c}\text { Escore do } \\
30 \\
\text { Trimestre/97 }\end{array}$ & $\begin{array}{c}\text { Escore do } \\
40 \\
\text { Trimestre/97 }\end{array}$ \\
\hline Argentina & $\mathrm{I}=\mathrm{X}_{1}-0,0014 \mathrm{X}_{2}+0,0008 \mathrm{X}_{3}$ & 0,99957 & 0,99927 & 0,99940 \\
Brasil & $\mathrm{I}=\mathrm{X}_{1}-0,0120 \mathrm{X}_{2}+0,0042 \mathrm{X}_{3}$ & 1,01478 & 1,00892 & $1,01493^{*}$ \\
Chile & $\mathrm{I}=\mathrm{X}_{1}-0,2908 \mathrm{X}_{2}+0,0424 \mathrm{X}_{3}$ & 0,79923 & 0,74388 & 0,79100 \\
China & $\mathrm{I}=\mathrm{X}_{1}-0,0482 \mathrm{X}_{2}+0,0095 \mathrm{X}_{3}$ & 0,95945 & 0,95559 & 0,95569 \\
Colômbia & $\mathrm{I}=\mathrm{X}_{1}-0,5666 \mathrm{X}_{2}+0,8304 \mathrm{X}_{3}$ & 1,51150 & 1,31001 & 1,43312 \\
Rep. Tcheca & $\mathrm{I}=\mathrm{X}_{1}-1,1498 \mathrm{X}_{2}+0,4280 \mathrm{X}_{3}$ & 0,56468 & 0,32299 & 0,38661 \\
Hong-Kong & $\mathrm{I}=\mathrm{X}_{1}-0,0112 \mathrm{X}_{2}+0,0064 \mathrm{X}_{3}$ & 0,99734 & 0,99547 & 0,99483 \\
Indonésia & $\mathrm{I}=\mathrm{X}_{1}-1,4611 \mathrm{X}_{2}+1,1158 \mathrm{X}_{3}$ & 1,58331 & 1,16383 & $1,60693^{*}$ \\
Jordânia & $\mathrm{I}=\mathrm{X}_{1}-0,0028 \mathrm{X}_{2}+0,0169 \mathrm{X}_{3}$ & 1,01644 & 1,01353 & 1,01347 \\
Coréia & $\mathrm{I}=\mathrm{X}_{1}-0,5960 \mathrm{X}_{2}+0,6376 \mathrm{X}_{3}$ & 1,58150 & 1,10196 & 1,57115 \\
Malásia & $\mathrm{I}=\mathrm{X}_{1}-1,2136 \mathrm{X}_{2}+1,7778 \mathrm{X}_{3}$ & 2,18410 & 1,95115 & 2,18367 \\
México & $\mathrm{I}=\mathrm{X}_{1}-0,2842 \mathrm{X}_{2}+0,1970 \mathrm{X}_{3}$ & 0,93888 & 0,83591 & 0,91683 \\
Peru & $\mathrm{I}=\mathrm{X}_{1}-0,2090 \mathrm{X}_{2}+0,4209 \mathrm{X}_{3}$ & 1,26295 & 1,20558 & 1,19659 \\
Filipinas & $\mathrm{I}=\mathrm{X}_{1}-0,5427 \mathrm{X}_{2}+0,4427 \mathrm{X}_{3}$ & 1,32019 & 1,18965 & 1,27474 \\
Polônia & $\mathrm{I}=\mathrm{X}_{1}-0,3745 \mathrm{X}_{2}+0,6076 \mathrm{X}_{3}$ & 1,36193 & 1,31665 & 1,24134 \\
Cingapura & $\mathrm{I}=\mathrm{X}_{1}-0,6462 \mathrm{X}_{2}+1,0360 \mathrm{X}_{3}$ & 1,56579 & 1,45359 & $1,58879 *$ \\
Sri Lanka & $\mathrm{I}=\mathrm{X}_{1}-0,0646 \mathrm{X}_{2}+0,1031 \mathrm{X}_{3}$ & 1,07509 & 1,03333 & 1,04959 \\
Tailândia & $\mathrm{I}=\mathrm{X}_{1}-1,7095 \mathrm{X}_{2}+1,1641 \mathrm{X}_{3}$ & 1,16183 & 1,15010 & 0,83143 \\
Turquia & $\mathrm{I}=\mathrm{X}_{1}-0,2473 \mathrm{X}_{2}+0,6118 \mathrm{X}_{3}$ & 1,61418 & 1,51533 & 1,55599 \\
Venezuela & $\mathrm{I}=\mathrm{X}_{1}-1,9473 \mathrm{X}_{2}+1,0284 \mathrm{X}_{3}$ & 0,92790 & $-0,07089$ & 0,24564 \\
\hline & & & & \\
& & & & \\
& & & &
\end{tabular}

Nota: ${ }^{*}=$ Países que sofreram pressão cambial, isto é, escores de crise $>\bar{I}+2 \sigma$. 
Embora o indicador proposto por Goldstein, Kaminsky e Reinhart (2000) não tenha classificado os principais protagonistas da crise asiática como aqueles que sofreram pressão cambial, exceto a Indonésia, não podemos considerá-lo como um indicador de menor ou pouca eficácia, pois os resultados podem se alterar com a mudança da periodicidade. $O$ ponto realmente relevante é que tal metodologia classifica os países que sofrem pressão cambial de forma arbitraria. Os próprios autores (GOLDSTEIN, KAMINSKY \& REINHART, 2000, p. 19) reconhecem que poderia se considerar como limite para classificar países que sofrem turbulência no mercado cambial escores com valores superiores a três desvios padrões além da média.

\section{CONCLUSÕES}

Ao comparamos a metodologia de mensuração de pressão cambial utilizada por Goldstein, Kaminsky e Reinhart (2000) com a nossa pudemos constatar vantagens e desvantagens de ambas as partes. Entre elas merece ser destacado que para estudos de pressão cambial de um país ou mesmo um pequeno grupo de países a metodologia de Goldstein, Kaminsky e Reinhart (2000) é a mais apropriada. Entretanto, para um estudo de caso considerando-se um grande número de países a metodologia por nós apresentada é a mais apropriada por ser mais parcimoniosa.

A utilização de um indicador agregado de pressão no mercado cambial como função da taxa de câmbio nominal, das reservas internacionais e das taxas de juros permitiu caracterizar os países mais vulneráveis às pressões cambiais e ataques especulativos, incluindo aqueles que possam ter sofrido ataques especulativos malsucedidos, como foi o caso do Brasil no $4^{\circ}$ trimestre de 1997.

A análise de conglomerados e sua validação via análise discriminante corroboram os resultados conhecidos. Países como Coréia, Filipinas, Indonésia, Malásia e Tailândia são confirmados como os principais protagonistas da crise cambial asiática ocorrida a partir de meados de 1997, como concluem Furman e Stiglitz (1998) e Radelet e Sachs (1998). Os países que sofreram maior pressão cambial no $3^{\circ}$ trimestre de 1997, em ordem decrescente, 
foram Tailândia, Indonésia e Filipinas, enquanto que no $4^{\circ}$ trimestre foram Indonésia, Coréia, Filipinas, Malásia, Tailândia, Brasil e Turquia.

APENDICE

Os dados que geraram a Tabela Al foram coletados no IFS (março de 1998) e representam observações do $3^{\circ}$ e $4^{\circ}$ trimestres de 1997 , respectivamente. A tabela contém observações trimestrais da taxa de câmbio nominal (Xl e $\mathrm{X} 2$ na linha $\mathrm{rf}$ ), observações trimestrais do nível de reservas internacionais (X3 e X4 na linha 1ld) e observações trimestrais da taxa de juros sobre depósitos (X5 e X6 na linha 601).

\section{TABELA AI - RAZÓES TRIMESTRAIS DAS TAXAS DE CÂMBIO NOMINAIS, DAS RESERVAS INTERNACIONAIS E DAS TAXAS DE JUROS NOMINAIS}

\begin{tabular}{lcccccc}
\hline Países & $\mathrm{X} 1$ & $\mathrm{X} 2$ & $\mathrm{X} 3$ & $\mathrm{X} 4$ & $\mathrm{X} 5$ & $\mathrm{X} 6$ \\
\hline Argentina & 1,000 & 1,000 & 0,981 & 1,153 & 0,982 & 1,235 \\
Brasil & 1,018 & 1,018 & 1,084 & 0,839 & 0,986 & 1,571 \\
Chile & 0,994 & 1,025 & 1,033 & 0,984 & 1,181 & 1,228 \\
China & 0,999 & 0,999 & 1,107 & 1,049 & 1,000 & 0,759 \\
Colômbia & 1,074 & 1,115 & 0,994 & 0,962 & 0,962 & 1,039 \\
R.Tcheca & 1,088 & 0,993 & 1,013 & 0,896 & 0,934 & 0,989 \\
Hong-Kong & 1,000 & 0,999 & 1,304 & 1,053 & 1,170 & 1,178 \\
Indonésia & 1,145 & 1,435 & 0,997 & 0,818 & 1,322 & 1,225 \\
Jordânia & 1,000 & 1,000 & 1,256 & 1,078 & 1,009 & 0,970 \\
Coréia & 1,008 & 1,273 & 0,892 & 0,670 & 0,981 & 1,094 \\
Malásia & 1,107 & 1,254 & 0,833 & 0,938 & 1,044 & 1,163 \\
México & 0,987 & 1,033 & 1,134 & 1,068 & 0,869 & 0,997 \\
Peru & 0,996 & 1,019 & 0,986 & 1,044 & 0,987 & 0,941 \\
Filipinas & 1,130 & 1,187 & 0,963 & 0,771 & 1,316 & 1,143 \\
Polônia & 1,084 & 1,011 & 1,019 & 1,033 & 1,010 & 1,015 \\
Cingapura & 1,037 & 1,072 & 0,959 & 0,922 & 1,000 & 1,073 \\
Sri Lanka & 1,012 & 1,016 & 1,200 & 0,968 & 0,954 & 0,928 \\
Tailândia & 1,275 & 1,231 & 0,913 & 0,915 & 1,232 & 1,000 \\
Turquia & 1,180 & 1,160 & 1,261 & 0,922 & 1,057 & 1,019 \\
Venezuela & 1,025 & 1,012 & 1,127 & 0,966 & 1,068 & 1,084 \\
\hline
\end{tabular}

Fonte: IFS - março (1998).

Nota: Variáveis identificadas na seção 4, p. 14. 
TABELA A2 - CLASSIFICAÇÃO DOS PAÍSES (3' TRIMESTRE DE 1997): FUNÇÃO DISCRIMINANTE LINEAR

\begin{tabular}{lcccc}
\hline Países & $\begin{array}{c}\text { Grupo de } \\
\text { Origem }\end{array}$ & Classificação & $\begin{array}{c}\text { Probabilidade de } \\
\text { Classificação do } \\
\text { Grupo } 1\end{array}$ & $\begin{array}{c}\text { Probabilidade de } \\
\text { Classificação do } \\
\text { Grupo } 2\end{array}$ \\
\hline Argentina & 1 & 1 & 1,0000 & 0,0000 \\
Brasil & 1 & 1 & 1,0000 & 0,0000 \\
Chile & 1 & 1 & 0,9999 & 0,0001 \\
China & 1 & 1 & 1,0000 & 0,0000 \\
Colômbia & 1 & 1 & 1,0000 & 0,0000 \\
Rep. Tcheca & 1 & 1 & 1,0000 & 0,0000 \\
Hong-Kong & 1 & 1 & 1,0000 & 0,0000 \\
Indonésia & 2 & 2 & 1,0000 & 0,0000 \\
Jordânia & 1 & 1 & 1,0000 & 0,0000 \\
Coréia & 1 & 1 & 1,0000 & 0,0000 \\
Malásia & 1 & 1 & 0,8710 & 0,1290 \\
México & 1 & 1 & 1,0000 & 0,0000 \\
Peru & 1 & 1 & 1,0000 & 0,0000 \\
Filipinas & 2 & 2 & 1,0000 & 0,0000 \\
Polônia & 1 & 1 & 0,9999 & 0,0001 \\
Cingapura & 1 & 1 & 1,0000 & 0,0000 \\
Sri Lanka & 1 & 1 & 1,0000 & 0,0000 \\
Tailândia & 2 & 2 & 1,0000 & 0,0000 \\
Turquia & 1 & 1 & 0,7880 & 0,2120 \\
Venezuela & 1 & 1,0000 & 0,0000 \\
\hline & & 1 & & \\
\hline
\end{tabular}


TABELA A3 - CLASSIFICAÇÃO DOS PAÍSES (4 TRIMESTRE DE 1997): FUNÇÃO DISCRIMINANTE LINEAR

\begin{tabular}{lcccc}
\hline Países & $\begin{array}{c}\text { Grupo de } \\
\text { Origem }\end{array}$ & Classificação & $\begin{array}{c}\text { Probabilidade de } \\
\text { Classificação do } \\
\text { Grupo 1 }\end{array}$ & $\begin{array}{c}\text { Probabilidade de } \\
\text { Classificação do } \\
\text { Grupo 2 }\end{array}$ \\
\hline Argentina & 1 & 1 & 1,0000 & 0,0000 \\
Brasil & 1 & 1 & 1,0000 & 0,0000 \\
Chile & 1 & 1 & 0,9999 & 0,0001 \\
China & 1 & 1 & 1,0000 & 0,0000 \\
Colômbia & 1 & 1 & 1,0000 & 0,0000 \\
Rep. Tcheca & 1 & 1 & 1,0000 & 0,0000 \\
Hong-Kong & 1 & 1 & 1,0000 & 0,0000 \\
Indonésia & 2 & 2 & 1,0000 & 0,0000 \\
Jordânia & 1 & 1 & 1,0000 & 0,0000 \\
Coréia & 1 & 1 & 1,0000 & 0,0000 \\
Malásia & 1 & 1 & 0,8710 & 0,1290 \\
México & 1 & 1 & 1,0000 & 0,0000 \\
Peru & 1 & 1 & 1,0000 & 0,0000 \\
Filipinas & 2 & 2 & 1,0000 & 0,0000 \\
Polônia & 1 & 1 & 0,9999 & 0,0001 \\
Cingapura & 1 & 1 & 1,0000 & 0,0000 \\
Sri Lanka & 1 & 1 & 1,0000 & 0,0000 \\
Tailândia & 2 & 2 & 1,0000 & 0,0000 \\
Turquia & 1 & 1 & 0,7880 & 0,2120 \\
Venezuela & 1 & 1 & 1,0000 & 0,0000 \\
\hline
\end{tabular}


TABELA A4 - CLASSIFICAÇÃO DOS PAÍSES NO $3^{\circ}$ TRIMESTRE DE 1997: FUNÇÃO DISCRIMINANTE (NÃO-PARAMÉTRICA) LINEAR SOBRE RANKS

\begin{tabular}{|c|c|c|c|c|}
\hline Países & $\begin{array}{c}\text { Grupo de } \\
\text { Origem }\end{array}$ & Classificação & $\begin{array}{l}\text { Probabilidade de } \\
\text { Classificação do } \\
\text { Grupo } 1\end{array}$ & $\begin{array}{c}\text { Probabilidade } \\
\text { de } \\
\text { Classificação } \\
\text { do Grupo } 2\end{array}$ \\
\hline Argentina & 1 & 1 & 0,9481 & 0,0519 \\
\hline Brasil & 1 & 1 & 0,9945 & 0,0055 \\
\hline Chile & 1 & $2^{*}$ & 0,3654 & 0,6346 \\
\hline China & 1 & 1 & 0,9873 & 0,0127 \\
\hline Colômbia & 1 & 1 & 0,9913 & 0,0087 \\
\hline R.Tcheca & 1 & 1 & 0,9989 & 0,0011 \\
\hline Hong-Kong & 1 & 1 & 0,8833 & 0,1167 \\
\hline Indonésia & 2 & 2 & 0,0594 & 0,9406 \\
\hline Jordânia & 1 & 1 & 0,9806 & 0,0194 \\
\hline Coréia & 1 & 1 & 0,9520 & 0,0480 \\
\hline Malásia & 1 & $2^{*}$ & 0,1945 & 0,8055 \\
\hline México & 1 & 1 & 0,9999 & 0,0001 \\
\hline Peru & 1 & 1 & 0,9374 & 0,0626 \\
\hline Filipinas & 2 & 2 & 0,0212 & 0,9788 \\
\hline Polônia & 1 & 1 & 0,8371 & 0,1629 \\
\hline Cingapura & 1 & 1 & 0,7665 & 0,2335 \\
\hline Sri Lanka & 1 & 1 & 0,9996 & 0,0004 \\
\hline Tailândia & 2 & 2 & 0,0288 & 0,9712 \\
\hline Turquia & 1 & 1 & 0,9384 & 0,0616 \\
\hline Venezuela & 1 & 1 & 0,8122 & 0,1878 \\
\hline
\end{tabular}

Nota: $\left({ }^{*}\right)=$ Observação classificada erroneamente. 
TABELA A5 - CLASSIFICAÇÃO DOS PAÍSES NO $4^{\circ}$ TRIMESTRE DE 1997: FUNÇÃO DISCRIMINANTE (NÃO-PARAMÉTRICA) LINEAR SOBRE RANKS

\begin{tabular}{lcccc}
\hline Países & $\begin{array}{c}\text { Grupo de } \\
\text { Origem }\end{array}$ & Classificação & $\begin{array}{c}\text { Probabilidade de } \\
\text { Classificação do } \\
\text { Grupo 1 }\end{array}$ & $\begin{array}{c}\text { Probabilidade de } \\
\text { Classificação do } \\
\text { Grupo 2 }\end{array}$ \\
\hline Argentina & 1 & 1 & 0,9870 & 0,0130 \\
Brasil & 2 & 2 & 0,0189 & 0,9811 \\
Chile & 1 & 1 & 0,7777 & 0,2223 \\
China & 1 & 1 & 0,9980 & 0,0020 \\
Colômbia & 1 & 1 & 0,7028 & 0,2972 \\
R. Tcheca & 1 & $2^{*}$ & 0,1908 & 0,8092 \\
Hong-Kong & 1 & 1 & 0,9864 & 0,0136 \\
Indonésia & 2 & 2 & 0,0134 & 0,9866 \\
Jordânia & 1 & 1 & 0,9981 & 0,0019 \\
Coréia & 2 & 2 & 0,0063 & 0,9937 \\
Malásia & 2 & 2 & 0,3373 & 0,6627 \\
México & 1 & 1 & 0,9970 & 0,0030 \\
Peru & 1 & 1 & 0,9950 & 0,0050 \\
Filipinas & 2 & 2 & 0,0152 & 0,9848 \\
Polônia & 1 & 1 & 0,9476 & 0,0524 \\
Cingapura & 1 & $2^{*}$ & 0,2351 & 0,7649 \\
Sri Lanka & 1 & 1 & 0,9696 & 0,0304 \\
Tailândia & 2 & 2 & 0,1805 & 0,8195 \\
Turquia & 2 & 2 & 0,2391 & 0,7609 \\
Venezuela & 1 & 1 & 0,6582 & 0,3418 \\
\hline
\end{tabular}

Nota: $\left({ }^{*}\right)=$ Observação classificada erroneamente.

\section{REFERÊNCIAS BIBLIOGRÁFICAS}

BIS - Bank For International Settlements. 68 ${ }^{\text {th }}$ Annual Report. 1998.

BLANCO, Herminio; GARBER, Peter M. Recurrent devaluation and speculative attacks on the Mexican peso. Journal of Political Economy, n. 4, p. 148-66, 1986.

CHATFIELD, C.; COLLINS, A. J. Introduction to multivariate analysis. Londres: Chapman and Hall, 1980.

CONOVER, W. J. Practical nonparametric statistics. Third Edition. John Wiley \& Sons, Inc. 1999. 
CUMBY, Robert E.; WIJNBERGEN, Sweder van. Financial policy and speculative runs with a crawling peg: Argentina, 1978-1981. Journal of International Economics, n. 27, p. 111-127, 1989.

EICHENGREEN, B.; ROSE, Andrew K; WYPLOSZ, C. Exchange market mayhem: the antecedents and aftermath of speculative attacks. Economic Policy, n. 21, p. 249-312, outubro 1995.

EVERITT, B. S.; DER, G. A handbook of statistical analyses using SAS. Nova York: Chapman \& Hall, 1996.

EVERITT, B. S.; DUNN, G. Applied multivariate data analysis. $2^{\circ} \mathrm{ed}$. Londres: Arnold, 2001.

FRANKEL, J.; ROSE, Andrew K. Currency crashes in emerging markets: empirical indicators. NBER Working Paper, nr. 5437, Cambridge, Massachusetts: National Bureau of Economic Research, Janeiro 1996.

FURMAN, J.; STIGLITZ, J. Economic crises: evidence and insights from east Asia. Brookings Papers on Economic Activity, 2, p. 1-133, 1998.

GIRTON, L.; ROPER, D. A monetary model of exchange market pressure applied to the postwar Canadian experience. The American Economic Review, v. 67, n. II, p. 537-548, 1977.

GOLDBERG, Linda S. Predicting exchange rate crises. Mexico revisited. Journal of International Economics, n. 3, p. 413-430, 1994.

GOLDFAJN, Ilan; VALDÉS, Rodrigo O. Are currency crises predictable? IMF Working Paper. Washington: International Monetary Fund, n. 159, 1997.

GOLDSTEIN, M.; KAMINSKY, G. L.; REINHART, C. M. Assessing financial vulnerability - an early warning system for emerging markets. Washington: Institute for International Economics, 2000.

IFS. International Financial Statistics. Março 1998.

JOHNSTON, Richard A.; WICHERN, Dean W. [1982]. Applied multivariate statistical analysis. 3a. ed. Nova Jersey: Prentice-Hall, Inc., 1992.

KAMINSKY, G.; LIZONDO, S.; REINHART, Carmen M. Leading indicators of currency crises. IMF Working Paper. Washington: International Monetary Fund, n. 79, julho 1997.

KAMINSKY, G.; REINHART, Carmen M. The twin crises: the causes of banking and balance-of-payments problems. The American Economic Review, v. 89, n. 3, p. 473-500, 1999.

MANLY, Bryan F. J. [1986] Multivariate statistical methods - a primer. Londres: $2^{a}$ ed., Chapman \& Hall, 1994.

MELICK, William R. Estimation of speculative attacks models: Mexico yet again. IMF Working Paper.Basle: Bank for International Settlements n. 36, 1996. 
MIRANDA, Mauro C. Crises cambiais e ataques especulativos no Brasil janeiro de 1982 a janeiro de 1999. 1999. Dissertação (Mestrado em Economia), UNB, Brasília.

OTKER, I.; PAZARBASIOGLU, C. Speculative attacks and currency crises: the Mexican experience. IMF Working Paper. Washington: International Monetary Fund, n. 112, 1995.

RADELET, S.; SACHS. The east Asian financial crisis: diagnosis, remedies, prospects. Brookings Papers on Economic Activity, 1, p. 1-90, 1998.

TREUHERZ, Rolf M. Manual das crises para paises emergentes. São Paulo: Editora Futura, 2000

WEYMEARK, D. A general approach to measuring exchange market pressure. Oxford Economic Papers, n. 50, p. 106-121, 1998.

WORLD ECONOMIC OUTLOOK. Financial crises - causes and indicators. International Monetary Fund, abril 1999.

Os autores agradecem a José Roberto Novaes de Almeida (UnB), Maria Luiza Falcão Silva (UnB), Geraldo Sant'ana de Camargo Barros (ESALQ) e aos pareceristas anônimos pelas contribuiçóes e pertinentes comentários. A responsabilidade pelos erros e omissões deste trabalho cabem exclusivamente aos autores.

tito@pos.ucb.br

mauriciopinto@brturbo.com

Geraldo.Souza@embrapa.br

(Recebido em agosto de 2002. Aceito para publicação em agosto de 2003). 